\title{
Archimedes' Principle and the Concept of Gravitation
}

\author{
Giancarlo Cavazzini ${ }^{1}$ \\ ${ }^{1}$ CNR Istituto di Geoscienze e Georisorse, Padova, Italy \\ Correspondence: Giancarlo Cavazzini, CNR Istituto di Geoscienze e Georisorse, Via G. Gradenigo 6, 35131, \\ Padova, Italy. Tel: 39-049-827-9137. E-mail: giancarlo.cavazzini@igg.cnr.it
}

Received: September 27, 2019

Accepted: October 26, 2019 Online Published: November 30, 2019

doi:10.5539/apr.v11n6p9

URL: http://dx.doi.org/10.5539/apr.v11n6p9

\begin{abstract}
Simple experimental evidence shows that the current physical interpretation of the phenomenon of a solid material body floating on the surface of a liquid body - known as 'Archimedes' Principle' - is not correct. As this interpretation is a consequence of the assumption that a volume of material, when immersed in a fluid, does not 'lose' its 'weight', what we believe of gravitational mechanical action is also not correct.

A material volume immersed in a fluid is currently believed to be subjected to two mechanical actions, 'gravitational mechanical action' or 'weight of the material volume' and 'Archimedes' force'. This is not in fact correct: the material volume is subjected to only one mechanical action, proportional to volume and to the difference in density between the matter of the material volume and that of the fluid.

We propose to call this mechanical action the 'weight of the material volume in that fluid', ceteris paribus. At the Earth's surface, floating of a solid material volume B on the surface of a liquid volume is the result of the concurrent action of two 'weights', the 'weight in air' of the part of volume B which is immersed in air, directed downwards, and the 'weight in the liquid' of the part of volume B which is immersed in the liquid, directed upwards.
\end{abstract}

Keywords: Archimedes' Principle, Floating, Weignt, Gravitation

\section{Introduction}

We currently believe that the intensity of the mechanical gravitational action generated on a material body by a second material body near the first body depends only on the 'masses' of the two bodies and on the distance between them. We believe that this mechanical action is independent of the matter of the body in which the two bodies are (partially or completely) immersed.

For example, let us consider a solid material body completely immersed in a liquid. We believe (in fact, we assume) that the gravitational action generated on the material body by the planet on which we live is equal to the gravitational action generated by the planet on the material body if that body is completely immersed in a vacuum. We believe that the gravitational action of a material body on a second body is only attractive (Mach, 1912), and that this action does not change in intensity whatever the fluid matter the bodies are immersed in, because it depends only on the 'masses' of the bodies involved, and, inversely, on the square of the distance between them (Mach, 1912).

It was the great physicist Isaac Newton who was the first to demonstrate that mechanical actions (which he called 'forces') between the Sun and other bodies in the Solar System may be interpreted in terms of reciprocal attraction. He also showed that the intensities of these mechanical actions depend on the 'mass' of the Sun and the 'masses' of the respective bodies and, inversely, on the square of the distance between the Sun and the other bodies (Newton, 1687).

In his famous experiment to determine the value of the 'gravitational constant', Henry Cavendish confirmed the dependence of gravitational mechanical action on the masses of the bodies involved and on the distance between them (Cavendish, 1798). Cavendish interpreted the process in terms of reciprocal attraction, although Newton himself did not believe gravitation was really an attraction at a distance between material bodies (Newton, 1693).

Calculating mass of a material body, however, means measuring volume and density of the matter which constitutes the body. Density is obtained by measuring the intensity of the mechanical action of which is home to a standard volume of the matter of the body, ceteris paribus. Ceteris paribus, however, the intensity of the 
observed mechanical action changes as the matter in which the body is immersed changes.

Let us consider a material body, and assume density of the matter of the body higher than density of water. All the other conditions being the same, if the material body is immersed in water the intensity of the observed mechanical action is different from intensity we measure in the case the body is immersed in a vacuum. In the case the material body is immersed in water, the intensity is lower.

This change in intensity is clearly determined by immersion in the fluid matter. However, it is not clear what this physically means. In fact, two interpretations can be given.

The first interpretation is that the change in intensity of the observed mechanical action represents a change in intensity of the mechanical action which we observe when the body is immersed in a vacuum. According to this interpretation, the material body is home to one mechanical action, the intensity of which changes.

The second interpretation is that the mechanical action we observe when the body is immersed in a vacuum does not change in intensity when the body is immersed in the fluid. The presence of a second concurrent mechanical action, opposite to the first one, is therefore necessary to explain the decrease in intensity when the body is immersed in the fluid matter. This is the current physical interpretation.

This shows that the belief that gravitational action is only attractive, and that its intensity does not change because it depends only on the masses of the bodies involved and on the distance between them is crucial, because it affects the physical interpretation of the phenomenon of floating - known as "Archimedes' Principle".

This Principle (Archimedes; Halliday et al., 2001) is perhaps the most important law in hydrostatics, at the base of the concept of Isostasy in geophysics (i.e., the relationship of floating of the Earth's crust on the Earth's mantle). It is still debated, because some aspects have not yet been exhaustively understood (Bierman \& Kincanon, 2003; Brown, 1999; Graf, 2004; Jones \& Gordon, 1979; Mohazzabi, 2017; Ray \& Johnson, 1979; Sears, 1963; Taibu, 2015; Taibu et al., 2015).

According to Archimedes' Principle, at the Earth's surface the mechanical behaviour of a material body completely immersed in a fluid body is interpreted as the result of the simultaneous action of two mechanical actions on that body. One is 'gravitational action' or the 'weight of the body', due to 'gravitational interaction' of the material body with the planet on which we live. This action is directed downwards. The second action is 'Archimedes' force' (or 'buoyancy'), believed to be due to the fluid in which the body is immersed: it is directed upwards.

It is believed that this physical interpretation was demonstrated according to the 'Principle of virtual work' (Leroy, 1985). However, this demonstration is weak from the epistemological viewpoint, as it involves assumptions and definitions, and is also considered with serious suspicion, because it does not explain the observed decrease in the weight of bodies which, if immersed in fluid bodies, sink to the bottom of their respective containers (Mohazzabi, 2017; Taibu, 2015; Taibu et al., 2015).

Let us examine the current interpretation. In the case of a material body completely immersed in a fluid body, the existence of 'Archimedes' force' is clearly based on the correctness of the hypothesis about gravitational action. If, as is believed at the present time, we assume that gravitational action exerted by a material body on a second material body depends only on the masses of those bodies and on the distance between them, this action does not change in intensity or direction, irrespective of the kind of fluid in which that body is immersed. Thus, in order to explain the observed change in the mechanical behavior of the material body with respect to such behavior in a vacuum, the existence of an opposite mechanical action on the body, variable in intensity, is necessary. The existence of Archimedes' force is therefore conditional on the correctness of the hypothesis on gravitational action.

Thus, if we prove that the observed mechanical behavior of the body is due to the concurrent action on it of 'weight' and 'Archimedes' force' as we have respectively defined them, the gravitational action on the body may be proportional to the mass of the body, unchanged, no matter in what the fluid body is immersed. Instead, if we prove that the behavior of the body cannot be due to the concurrence of such two mechanical actions, we must question the hypothesis about gravitational action. Therefore, proving that the current interpretation of the phenomenon studied by Archimedes is correct or incorrect is crucial to the debate on gravitation.

The question about the existence or otherwise of Archimedes' force in the case of bodies completely immersed in fluid bodies that sink and reach the bottom of their containers was first raised by Bierman and Kincanon (2003), and later, more explicitly, by Graf (2004). They emphasized that in those cases Archimedes did not refer to buoyancy at all, but they did not answer the question. 
Providing such an answer is the aim of this work: we propose a simple line of reasoning to show that the current hypothesis of gravitational action implies an incorrect physical interpretation of the phenomenon of floating, i.e., it is incorrect. This line of reasoning is a new and better discussed version of that proposed by Cavazzini (2018).

\section{Error in Current Physical Interpretation of Floating of Bodies}

1. Let us consider a solid material volume, a free rigid, homogeneous solid body B immersed in a vacuum (Figure 1(a)). We observe that the material volume is not in mechanical equilibrium. We give the reason for this: the material volume 'is subjected to' or, rather, 'is home to' a mechanical action which tends to move the material volume downwards. The mechanical action is due to a physical process which we believe is the expression of an 'interaction' between the material body and the planet on which we live. We call this physical process 'gravitation', and the mechanical action the 'gravitational action on the body' or, simply, the 'weight of the body'.

As usual, we graphically represent the mechanical action with an arrow, positioned in the center of the material volume. The direction of the arrow is the direction of the mechanical action, and the length of the arrow is proportional to intensity of the mechanical action.

2. Let us now consider the material body floating on the surface of a liquid. In this case, although it is free, the body is not moved downwards or upwards, i.e., it is in mechanical equilibrium (Figure 1(b)).

3. If we assume that the material body is still home to a downward-directed mechanical action, the observed equilibrium must result from the simultaneous action, 'on the body', of an opposite mechanical action, of 'intensity' equal to the intensity of the action directed downwards.

4. The only difference between the case of the body immersed in a vacuum and that of the body floating on the surface of the liquid is that, in the latter situation, a part $\mathrm{v}$ of the volume of the body is immersed in the liquid. Therefore, the mechanical upward-directed action which causes the equilibrium of floating originates from immersion in the liquid of part $\mathrm{v}$ of the material volume.

5. Unfortunately, equilibrium does not supply us with any information about the intensity of these two opposite mechanical actions. However, two hypotheses can be proposed.

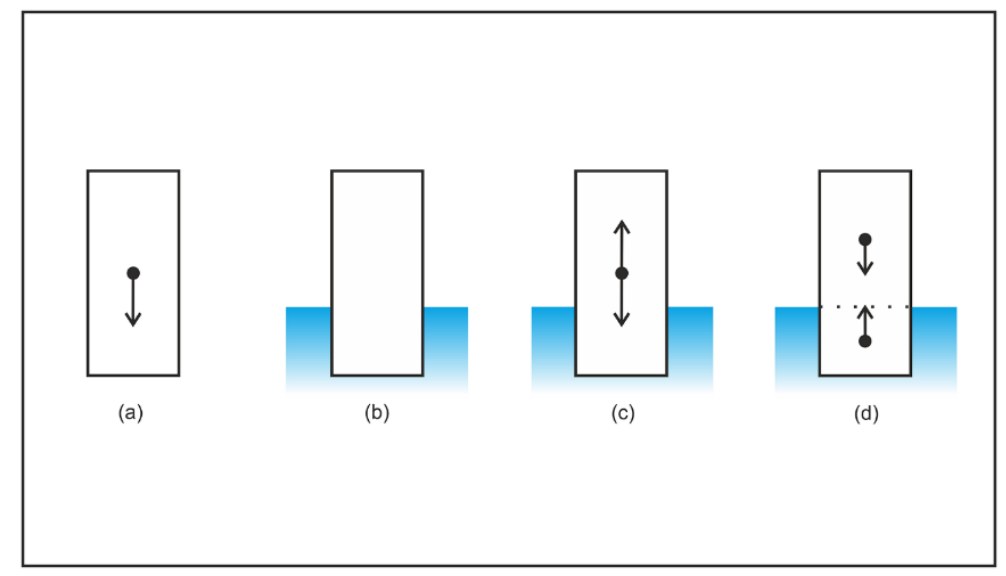

Figure 1. (a) A solid material volume completely immersed in a vacuum is in mechanical disequilibrium. (b) Material volume floating on surface of liquid is in mechanical equilibrium. Part of volume is immersed in liquid, remaining part is immersed in vacuum. (c) Current mechanical interpretation of equilibrium of floating. (d)

Second possible mechanical interpretation of equilibrium of floating

6. First hypothesis: the downward-directed mechanical action is equivalent in intensity to that which we observe when the material volume is completely immersed in a vacuum (Point 1.). This is equivalent to hypothesizing that the mechanical gravitational action between the planet on which we live and the material volume does not change, in whatever fluid body the material volume is immersed. This hypothesis, shown in Figure 1(c), leads to current interpretation: the equilibrium of floating is given by the concurrence (existence) of a mechanical action equivalent and opposite to gravitational mechanical action, the 'Archimedes' force'.

7. Second hypothesis: a volume of the matter which constitutes the material body, when immersed in the liquid, 'loses' its weight. By this, we mean that the volume, when immersed in the liquid, is no longer home to the 
downward-directed mechanical action of which it is home to if it is immersed in a vacuum. According to this hypothesis, the equilibrium of floating is given by the concurrence of the downward-directed action which is home to the part of the material body not immersed in the liquid (i.e. immersed in a vacuum), and of the upward-directed action which is home to the part of the material volume which is immersed in the liquid.

In this interpretation, shown in Figure 1(d), the intensity of the two mechanical actions causing the equilibrium is lower than in the current view.

8. Let us now consider a material volume floating on the surface of a liquid. The upward-directed mechanical action which gives rise to the equilibrium originates by immersion in the liquid of a part of the material volume (Point 4.). We observe that this material volume, immersed in the liquid to a greater extent than the part necessary to provide the equilibrium of floating, balances a certain load 'external' to itself (we assume to set the external material volume on top of the material volume). If the magnitude of this external load increases, we observe progressive immersion of the material volume in the liquid, and a maximum external load is balanced if the material volume is completely immersed.

9. Clearly, the maximum external load which is balanced is that which exactly determines complete immersion of the material volume in the liquid; however, no part of it is immersed in the liquid. A load that, at equilibrium, determines the complete immersion of the material volume, and, in turn, partly immerses itself in the liquid, is not balanced by the upward-directed mechanical action which originates from complete immersion of the material volume. Such a load partially balances itself by immersion in the liquid of part of its own volume.

10. Let us now examine the equilibrium of floating of material volume B (Figure 2(a), in which $\mathrm{v}$ is the part immersed in the liquid and $\mathrm{C}$ is the part not immersed. We now cut material volume $\mathrm{B}$ into two parts, $\mathrm{v}$ and $\mathrm{C}$ (Figure 2(b)). The external load which is balanced by the upward-directed mechanical action which originates from complete immersion in the liquid of volume $\mathrm{v}$ is exactly the load represented by volume $\mathrm{C}$. If we consider volume $\mathrm{C}$, the external load balanced by the upward-directed mechanical action which originates from complete immersion in the liquid of this volume is the load represented by volume D (Figure 2(c)).

If immersed volumes $\mathrm{v}$ and $\mathrm{C}$ are composed of the same material, and if the respective external volumes $\mathrm{C}$ and $\mathrm{D}$ are also composed of the same material, a proportionality exists between the immersed volume and the external balanced volume. For the sake of simplicity, in Figure 2(b) - (e) all the volumes, whether immersed or not, are composed of the same material.

11. Since this proportionality, a volume equivalent to the sum of two volumes, when immersed in the liquid balances an external volume/load which is the sum of the external volumes/loads respectively balanced by the two volumes when they are immersed in the liquid separately. Volume B, which is the sum of volumes $\mathrm{v}$ and $\mathrm{C}$, when immersed in the liquid, balances an external load/volume A, which is the sum of external loads C and D balanced, respectively, by volumes $\mathrm{v}$ and $\mathrm{C}$ when the volumes are immersed in the liquid separately (Figure 2 (d)).

12. If volumes $\mathrm{v}$ and $\mathrm{C}$ are placed one on top of the other, stacked together in the liquid as shown in Figure 2(e), they balance a total external load equivalent to the sum of the external loads which the two volumes balance separately. A comparison of Figure 2 (d) and (e) shows that these two volumes $\mathrm{v}$ and $\mathrm{C}$ stacked together in the liquid 'work' as a single body, equivalent to the sum of the two volumes (volume B, see Point 11.). The comparison, vice-versa, also shows that volume B immersed in the liquid can be ideally considered as the sum of two or more separate volumes, and that each of these volumes proportionally contributes to balancing external load A. In Figure 2(d), a part $\mathrm{v}$ of volume $\mathrm{B}$ balances a part $\mathrm{C}$ of external load $\mathrm{A}$, and the remaining part $\mathrm{C}$ of volume B balances a part D of the external load A. In other words, in Figure 2(d), the upward-directed action which takes origin due to immersion in the liquid of a part $\mathrm{v}$ of volume B contributes to balancing external load A. 


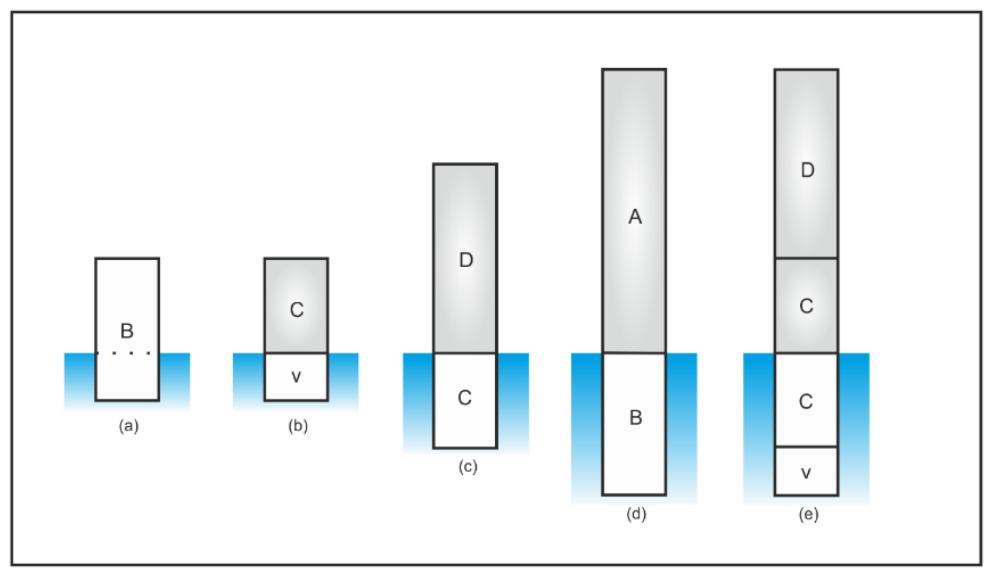

Figure 2. (a) Solid material volume B floating on surface of liquid. Part $\mathrm{v}$ of volume B is immersed in liquid; part $\mathrm{C}$ is immersed in vacuum. (b) Volume $\mathrm{B}$ is divided into two parts, $\mathrm{v}$ and $\mathrm{C}$. C is external load balanced by upward-directed mechanical action originating from immersion in liquid of volume v. (c) D is external load balanced by upward-directed action originating from immersion in liquid of volume C. (d) Volume A is external load balanced by upward-directed mechanical action originating from immersion in liquid of volume $\mathrm{B}$. Volume $\mathrm{B}$ is sum of volumes $\mathrm{v}$ and $\mathrm{C}$; balanced external load $\mathrm{A}$ is sum of external loads $\mathrm{C}$ and $\mathrm{D}$, respectively balanced by upward-directed actions originating from immersion in liquid of volume $\mathrm{v}$ and volume $\mathrm{C}$, when the latter are immersed in liquid separately. (e) Volumes $\mathrm{v}$ and $\mathrm{C}$, stacked together and immersed in liquid, balance an external load which is sum of external loads which two volumes respectively balance when immersed in liquid separately

13. At this point, we have some essential information: in Figure 2(d), in which volume B is immersed in the liquid, a part $\mathrm{v}$ of this volume contributes to balancing the external load represented by volume $\mathrm{A}$.

14. Let us now examine the floating of volume B in Figure 2(a) according to the current hypothesis on gravitational mechanical action (Point 6.). According to this hypothesis, a volume of the matter which constitutes the body, when immersed in the liquid, becomes home to an upward-directed mechanical action, but it is still home to the gravitational action of the planet Earth. In other words, that part of the volume of the body which is immersed in the liquid does not 'lose' its weight. According to this assumption, part $\mathrm{v}$ of volume B does not lose its weight. If this is true, in order to cause equilibrium, volume $\mathrm{v}$ immersed in the liquid must be home to an upward-directed mechanical action which balances not only the weight of the part of volume B which is not immersed in the liquid (volume $\mathrm{C}=\mathrm{B}-\mathrm{v}$, immersed in vacuum), but also that of volume v itself. In other words, according to the current hypotheses on gravitational action, the upward-directed action which originates from immersion in the liquid of part $\mathrm{v}$ of volume $\mathrm{B}$ balances the weight of the whole body $\mathrm{B}$.

15. We now put an external volume on top of body B floating on the surface of the liquid. When we position this volume, a new situation of equilibrium takes place, due to immersion in the liquid of part of volume $\mathrm{B}$, in addition to volume $\mathrm{v}$. We choose the external volume so that, at equilibrium, no part of it is immersed in the liquid, whereas volume B is completely immersed. In other words, a new equilibrium occurs when part C of volume B is completely immersed, in addition to part v. Volume B is now completely immersed in the liquid, whereas no part of the volume/load external to volume B is immersed. Therefore, this volume/load is exactly the external load balanced by the upward-directed action which originates from complete immersion in the liquid of volume B (i.e., it is equivalent to load/volume A) (Figure 2(d)).

16. According to current hypotheses on gravitational action, in the case of equilibrium of floating of body $B$ alone, the upward-directed mechanical action which originates by immersion of part $\mathrm{v}$ of volume $\mathrm{B}$ balances the weight of whole body B (Figure 2(a), Point 14). Threfore, according to this hypothesis, when we position external load $\mathrm{A}$ on top of volume $\mathrm{B}$ (Point 15), and new equilibrium occurs by immersion in the liquid of part $\mathrm{C}$ of volume $\mathrm{B}$ in addition to volume $\mathrm{v}$, the weight of body $\mathrm{B}$ does not change, because part $\mathrm{C}$ of volume $\mathrm{B}$ does not lose its weight when immersed in the liquid. Therefore, according to the current hypothesis on gravitational action, the upward-directed mechanical action which originates from immersion in the liquid of part $\mathrm{v}$ of volume $\mathrm{B}$ continues to balance the weight of the whole body B, and does not/cannot contribute towards balancing external load A. Only part C of volume B balances external volume A.

17. Nevertheless, we have demonstrated (Point 12., comparison between Figure 2(d) and 2 (e)) that the 
upward-directed mechanical action which originates from immersion in the liquid of part $\mathrm{v}$ of volume $\mathrm{B}$ contributes to balancing external volume/load A (the action balances part $\mathrm{C}$ of external volume A). Therefore, observation unambiguously indicates that the current interpretation of the equilibrium of floating is incorrect.

18. Since this interpretation is a consequence of current hypotheses on gravitational mechanical action, we infer that this hypothesis is incorrect.

\section{Discussion}

We have proved that a contradiction exists between observed facts and the current mechanical interpretation of the equilibrium of floating. Comparison of Figure 2(d) and 2(e) unambiguously indicates that in Figure 2(d) external load A is proportionally balanced by the upward-directed mechanical action which originates from immersion in the liquid of part $\mathrm{v}$ of volume $\mathrm{B}$, and by the upward-directed action which originates by immersion in the liquid of part $\mathrm{C}$ of volume B. In other words, in Figure 2(d), the upward-directed mechanical action which originates from immersion in the liquid of part $\mathrm{v}$ of volume $\mathrm{B}$ contributes towards balancing external load $\mathrm{A}$.

Instead, according to current physical interpretations, in equilibrium of floating of body B alone (Figure 2(a)), the upward-directed mechanical action which originates from complete immersion in the liquid of part $\mathrm{v}$ of volume B balances the weight of the whole of volume B. This interpretation is due to current hypothesis on gravitational mechanical action, because the part of volume B which is immersed in the liquid, i.e., volume v, does not 'lose' its weight. Accordingly, when external volume A is positioned on top of volume B and a new equilibrium occurs by immersion in the liquid of part $\mathrm{C}$ of volume $\mathrm{B}$ in addition to volume v (Figure 2(d)), part $\mathrm{C}$ of volume $\mathrm{B}$ does not lose its weight either. Therefore, according to current hypothesis on gravitational action, in Figure 2(d) the upward-directed mechanical action which originates from immersion in the liquid of part $\mathrm{v}$ of volume $\mathrm{B}$ must continue to balance the weight of the whole volume $\mathrm{B}$, and cannot contribute to balancing external load A. As this physical interpretation of equilibrium of floating is a consequence of the current hypothesis on gravitational mechanical action, we infer that the hypothesis is incorrect.

We infer instead that a volume of the matter constituting body B, when immersed in the liquid, is no longer home to the downward-directed mechanical action observed when the volume is immersed in a vacuum (i.e., the volume, when immersed in the liquid, 'loses' its weight). Accordingly, in the case of body B which floats alone on the surface of the liquid (Figure 2(a)), volume $\mathrm{v}$ is home to only one mechanical action, directed upwards. Since volume $\mathrm{v}$ (immersed in the liquid) has lost its weight, this upward-directed mechanical action does not balance the weight of the whole of body B, but only the weight of that part of volume B which is not immersed in the liquid (i.e., the weight of volume $\mathrm{C}=\mathrm{B}-\mathrm{v}$ ).

When we set external volume A on top of volume B, and part C of volume B is immersed in the liquid in addition to part $\mathrm{v}$, volume $\mathrm{C}$ also loses its weight, becoming home to only one, upward-directed, mechanical action. In this case, the mechanical action arising from immersion in the liquid of volume $\mathrm{v}$ no longer balances the weight of volume $\mathrm{C}$. Therefore, this upward-directed mechanical action, unchanged in intensity, contributes towards balancing the weight of a part - part C - of volume A. This interpretation matches observations.

In short, the upward-directed mechanical action which originates from immersion in the liquid of part $\mathrm{v}$ of volume B contributes to balancing load A because volume v, when immersed in the liquid, 'loses' its weight.

\section{Intensity of Mechanical Action}

We have shown that, at the Earth's surface, a solid body completely immersed in a fluid body is not home to two mechanical actions - one due to 'gravitational interaction' of the body with the planet, and 'Archimedes' force', as they are currently defined - but is home to only one mechanical action. We can now formulate the intensity of this mechanical action.

$\boldsymbol{A}$. Let us consider a material volume B immersed in a vacuum. We observe that this volume, matter 1, is home to a mechanical action. We now consider a second equivalent volume B, composed of matter differing from that of the first volume (matter 2). In general, all other conditions remaining unchanged, first volume B, in a vacuum, is home to a mechanical action of intensity differing from that of second volume B. For example, we may assume first volume B composed of solid material (matter 1 = copper), and second volume B composed of liquid material (matter 2 = water). In this case, in a vacuum, first volume B is home to a mechanical action greater than the mechanical action which, in a vacuum, is home to second material volume B. Alternatively, we may assume that matter 1 is wood: in this case, in a vacuum, first volume B is home to a mechanical action which is lower than the mechanical action which, in a vacuum, is home to second volume B.

Let us now consider a volume composed of matter 1, completely immersed in a material volume composed of matter 2. If, in a vacuum, the intensity of the mechanical action which is home to the volume composed of matter 
1 is equivalent to that of the mechanical action which is home to an equivalent volume composed of matter 2, the volume of matter 1 is not home to any observed mechanical action.

Instead, if the volume of matter 1, in a vacuum, is home to a mechanical action differing in intensity from that which, in a vacuum, is home to an equivalent volume composed of matter 2, we observe that the volume constituted by matter 1 , immersed in a volume of matter 2 , is home to a mechanical action.

Assuming matter 1 to be solid and matter 2 fluid (for example, liquid), if the volume of matter 1, in a vacuum, is home to a mechanical action higher than the action which, in a vacuum, is home to an equivalent volume of liquid matter, the solid volume, when immersed in the liquid matter, is home to a downward-directed action. Instead, if the solid volume in vacuum is home to a mechanical action lower than that which is home to an equivalent volume of the liquid matter, we observe that the solid volume, when immersed in the liquid, is home to an upward-directed mechanical action.

In other words, a relationship seems to exist between the direction of the mechanical action which is home to a material volume immersed in a fluid material and the sign of the difference in the value, between the material volume and the fluid material, of the property of the matter which determines the intensity of the mechanical action observed in a vacuum for equivalent volumes. If the sign of the difference in the value of the property between the material volume and the fluid material changes, the mechanical action which the material volume is home to reverses its direction. If the value of the difference in the value of the property between the matter of the material volume and the matter of the fluid is 0 , the material volume is not home to an obser4vable mechanical action. These observations suggest that the intensity of the mechanical action in question is proportional to the difference in the value of the property between the matter of the material volume and the matter of the fluid the material volume is immersed in.

$\boldsymbol{B}$. We now calculate the intensity of the upward-directed mechanical action of which part $v$ of volume $B$ becomes home when immersed in the liquid (Figure 2(a)).

Let us consider first body B completely immersed in air, which is home to a downward-directed mechanical action. We design a method to measure the 'intensity' of that mechanical action and, all other conditions remaining unchanged, we observe that the intensity of this action is proportional to the volume of the body. We can thus write ( $\mathrm{I}=$ Intensity, $\downarrow=$ downwards):

$$
I_{\downarrow}=B k_{1}
$$

where $k_{1}$ is a constant.

Therefore, all other conditions remaining the same, the downward-directed mechanical action which is home to volume $B-v$ of the matter of volume $B$, immersed in air, is $(B-v) k_{1}$.

The proportionality to volume also holds for the intensity of the downward-directed mechanical action of volume $V$ of a liquid. When immersed in air, the volume is home to a downward-directed mechanical action, the intensity of which is $V k_{2}$.

When body B floats on the surface of the liquid (Figure 2(a)), at equilibrium, a part $v$ of its volume is immersed in the liquid. Ceteris paribus, we observe - Archimedes observed (Proposition 5, Book I) - that volume $v$ is such that:

$$
B k_{1}=v k_{2}
$$

In other words, the downward-directed mechanical action which is home to volume $v$ of the liquid when that volume is immersed in air, is equal to the downward-directed mechanical action which body B is home to when this body is immersed in air.

As volume $B-v$, in air, is home to a downward-directed mechanical action

$$
(B-v) k_{1}=B k_{1}-v k_{1}
$$

from equation (2) we obtain:

$$
B k_{1}-v k_{1}=v k_{2}-v k_{1}
$$

and the intensity of the upward-directed mechanical action which is due to the immersion in the liquid of volume $v$ is therefore:

$$
I_{\uparrow}=-v\left(k_{1}-k_{2}\right)
$$


In Section $\boldsymbol{A}$. we have shown that the intensity of the mechanical action which is home to a volume of matter immersed in a volume of matter of a different kind is proportional to the difference in the value of the property of the matter which, ceteris paribus, determines the intensity of the mechanical action observed in a vacuum for an equivalent volume of matter. Thus, all other conditions remaining the same, $k_{1}$ and $k_{2}$ are respectively proportional to the difference in the value of this property.

In the case of a solid body immersed in air ( $p=$ property; $k=$ equals conditions):

$$
k_{1}=\left(p-p_{\text {air }}\right) k
$$

and, in the case of the liquid immersed in air:

$$
k_{2}=\left(p_{\text {liq }}-p_{\text {air }}\right) k
$$

Therefore:

$$
k_{1}-k_{2}=\left(p-p_{l i q}\right) k
$$

and the intensity of the upward-directed mechanical action is:

$$
I_{\uparrow}=-v\left(p-p_{\text {liq }}\right) k
$$

We call 'density' $(d)$ the property of the matter in relation to the intensity of the mechanical action which, in a vacuum, is home to the unit of volume in question. Therefore, the upward-directed mechanical action which originates from immersion in a liquid of solid volume $v$ is proportional to volume, and to the difference in density between the matter of both solid material and liquid material:

$$
I_{\uparrow}=-v\left(d-d_{l i q}\right) k
$$

If volume $B-v$ is immersed in air, the equilibrium of floating of body B is (omitting $k$ ):

$$
(B-v)\left(d-d_{\text {air }}\right)=-v\left(d-d_{\text {liq }}\right)
$$

$\boldsymbol{C}$. We call 'density' the property of matter which, ceteris paribus, determines the value of the intensity of the mechanical action which is home to the unit of volume of matter immersed in a vacuum. Since a volume of vacuum immersed in a vacuum is home to a mechanical action of intensity 0 , that vacuum has a value of the property of 0 . Therefore, if volume $\mathrm{B}-\mathrm{v}$ is immersed in a vacuum, the mechanical equilibrium of floating is:

$$
(B-v)(d-0)=-v\left(d-d_{l i q}\right)
$$

where value $v$ is different (higher) than value $v$ in equation (11).

It is important to note that, from a mathematical (algebraic) viewpoint, equation (12) can be reduced to the current formulation of the equilibrium of floating:

$$
B d=v d_{l i q}
$$

where, as currently defined in physics textbooks, quantity $B d$ is the weight of the whole body B and quantity $v d_{l i q}$ is the weight of the volume of liquid displaced.

Therefore, as emphasised by Cavazzini (2018), in this case where the part of material volume B not immersed in the liquid is immersed in a vacuum, formulas (12) and (13), although clearly not equivalent from a physical viewpoint, are equivalent from a quantitative viewpoint.

D. Material volume $\mathrm{V}$ of volume $V$ and density $d$ immersed in a vacuum is therefore home to a mechanical action of intensity:

$$
I_{\downarrow}=V(d-0) k=V d k=m k
$$

i.e., if the density of the matter constituting material volume $V$ is much higher than that of the fluid body in which $V$ is immersed, the value of the difference in density between the materials is close to that of the density of the matter constituting $V$ itself. In this case, the intensity of the mechanical action is proportional to the quantity $m$ which is the product between the volume of the body and the density of the matter of the body, i.e., the intensity of the mechanical action is proportional to what we have called the 'mass of the body'. However, this proportionality is not the rule, but is the result of particular conditions. For example, it is the case of celestial bodies immersed in a 'cosmic vacuum'. 


\section{Conclusions}

According to the current interpretation of the equilibrium of floating of a solid volume on the surface of a liquid volume, the part of the solid volume immersed in the liquid is home to an upward-directed mechanical action which equilibrates the weight of the whole solid volume. The basis of this interpretation is the assumption that the gravitational action of the planet on the solid volume, all other conditions remaining the same, does not change in intensity or direction, regardless of the fluid in which the solid volume is immersed. If this is true, the upward-directed action which is home to that part of the solid volume immersed in the liquid cannot contribute towards balancing external load A in Figure 2(d), because that part does not 'lose' its weight when it is immersed in the liquid.

Facts unambiguously indicate, however, that the upward-directed mechanical action which is home to the part immersed in the liquid of volume B (part v, Figure 2 (a)) contributes towards balancing the external load/volume A in Figure 2(d). This shows that the current physical interpretation of the equilibrium of floating is incorrect. Since the basis for this interpretation is the hypothesis about gravitational action, this indicates that this hypothesis is incorrect.

A material volume completely immersed in a fluid body is not, as currently believed, home to two mechanical actions - the 'gravitational force' or 'weight' of the material volume, directed downwards, and 'Archimedes' force', directed upwards, but to only one mechanical action.

We propose calling this mechanical action 'gravitational action on the material volume in that fluid', or the 'weight of the material volume in that fluid', ceteris paribus. According to this interpretation, all other conditions remaining the same, the weight of a material volume generally changes in intensity and direction if the matter of the fluid in which it is immersed also changes. For example, the weight of a piece of wood in air is directed downwards, whereas in water it is directed upwards. The (total) weight of the piece of wood is null if that wood is immersed in a fluid the matter of which has a density equal to that of the matter constituting it.

According to this definition of "weight", at the Earth's surface, the equilibrium of floating of a material body B on the surface of a liquid is the result of the concurrent action of the weight in air of volume B - v, directed downwards, and the weight in the liquid of volume, directed upwards (Figure 2(a) and 1(d)). The intensity of these two mechanical actions is lower than that of the mechanical actions which cause the equilibrium of floating according to current interpretation (Figure 1(d) vs. (1c), respectively).

Again, according to this definition of 'weight', gravitation may be interpreted not only as an 'attractive' 'force' but also as a 'repulsive' one (see the example of the change in the direction of movement of the piece of wood immersed in air and in water). However, attraction or repulsion are only apparent, because neither is the result of the process. The gravitational process moves a material volume in the direction in which the disequilibrium in density between the matter constituting the material volume and the matter constituting the fluid body in which the volume is immersed decreases. If moving the material volume leads to equilibrium in density between the matter constituting the volume and the matter constituting the fluid body in which the volume is immersed, the mechanical action on the surroundings ceases, because the total sum of the mechanical gravitational action on the material volume becomes null.

Therefore, if we define the weight of a material volume - i.e., gravitation - as proposed here, we can interpret this physical process according to its fundamental nature, i.e., its spontaneity. Gravitation is a spontaneous process and, like all other spontaneous processes in nature, originates from a disequilibrium, and tends to restore an equilibrium.

\section{Acknowledgments}

This work was supported by C.N.R Istituto di Geoscienze e Georisorse. The Author is very grateful to Ms. Gabriel Walton for her generous care in language revision, and to Nicola Michelon for having drawing figures.

\section{Conflict of interests}

The authors declare that there is no conflict of interests regarding the publication of this paper.

\section{References}

Archimedes of Syracuse. On floating bodies, Book I (in Italian, in Opere di Archimede; Torino, UTET, 1974).

Bierman, J., \& Kincanon, E. (2003). Reconsidering Archimedes' Principle. The Physics Teacher, 41, 340-344.

Brown, R. (1999). Weight - don't use the word at all. The Physics Teacher, 37, 241. 
Cavazzini, G. (2018). A new physical interpretation of Archimedes' Principle. Journal of Applied Mathematics and Physics, 6, 215-223.

Cavendish, H. (1798). Experiments to determine the density of the Earth. Transactions Royal Society of London, 88, 469-526. Retrieved from https://www.jstor.org/stable/106988

Graf, E. H. (2004). Just what did Archimedes say about buoyancy? The physics Teacher, 42, 296-299.

Halliday, D., Resnick, R., \& Walker, J. (2001). Fundamentals of Physics (6th ed.). New York, Wiley.

Jones, G. E., \& Gordon, W. P. (n. d.). Removing the buoyant force. The Physics Teacher, 17, 59-60.

Leroy, B. (1985). Archimedes' Principle: A simple derivation. European Journal of Physics, 6, 56.

Mach, E. (1912). Die Mechanikin ihrer Entwickelung Historisch-Kritish Dargestellt (7th ed.) (in Italian)

Mohazzabi, P. (2017). Archimedes' Principle revisited. Journal of Applied Mathematics and Physics, 5, 836-843.

Newton, I. (1687). Philosophiae Naturalis Principia Mathematica (1st ed.). London, Societatis Regiae ac Typis, Joseph Streater.

Newton, I. (1752). Four letters from Sir Isaac Newton to R. Bentley, containing some arguments in proof of a deity (1693). London, R. and J. Dodslet.

Ray, J. R., \& Johnson, E. (1979). Removing the buoyant force, a follow-up. The Physics Teacher, 17, 392-393.

Sears, F. W. (1963). Weight and weightlessness. The Physics Teacher, 1, 20-23.

Taibu, R. (2015). Terms vs, concepts - the case of weight. The Physics Teacher, 55, 34-35

Taibu, R., Rudge, D., \& Schuster, D. (2015). Textbooks presentations of weight: Conceptual difficulties and language ambiguities. Physical Review Education Research, 11, 1-20

\section{Copyrights}

Copyright for this article is retained by the author(s), with first publication rights granted to the journal.

This is an open-access article distributed under the terms and conditions of the Creative Commons Attribution license (http://creativecommons.org/licenses/by/4.0/). 